\title{
The Use of Multi-Element Aerosol Particles for Determining Temporal VARIATIONS IN TEMPERATURE AND ELECTRON DENSITY IN LASER-INDUCED PLASMAS IN SUPPORT OF QUANTITATIVE LIBS
}

\author{
Michael E. Asgill', Sebastian Groh², Kay Niemax³ , David W. Hahn ${ }^{1}$ \\ ${ }^{1}$ University of Florida, Department of Mechanical and Aerospace Engineering, Gainesville, FL 32611- \\ 6250 \\ ${ }^{2}$ Leibniz-Institut für Analytische Wissenschaften (ISAS), Bunsen-Kirchhoff-Strasse 11, 44139 Dortmund, \\ Germany \\ ${ }^{3}$ Federal Institute for Materials Research and Testing (BAM), Department 1: Analytical Chemistry and \\ Reference Materials, Richard-Willstaetter-Strasse 11, 12489 Berlin, Germany
}

\begin{abstract}
Quantitative laser-induced breakdown spectroscopy (LIBS) analysis operates on the assumption that the sample is completely dissociated and diffused within the highly energetic plasma on time-scales of analyte analysis, resulting in analyte emission ideally at the bulk plasma temperature and a signal that is linear with analyte mass concentration. However, recent studies focusing on aerosol analysis have found the heat and mass diffusion rates within laser-induced plasmas to be finite, resulting in particle-rich, locally perturbed areas within the hot bulk plasma. The goal of this study is to observe any related plasma differences, by calculating the bulk and local (i.e. analyte rich regions) plasma temperatures and electron density, to better understand the time frame of equilibrium between the local and bulk plasma properties. This study also seeks to determine whether the presence of large quantities of a matrix element can significantly alter the local plasma conditions, thereby generating matrix effects. We report the temporal profiles of particle-derived species, adding additional insight into the effect of local perturbation of plasma properties, with the conclusion that significant plasma residence (tens of microseconds) is necessary to minimize such effects.
\end{abstract}


The USE of Multi-Element Aerosol Particles for Determining Temporal Variations in TEMPERATURE AND ELECTRON DENSITY IN LASER-INDUCED PLASMAS IN SUPPORT OF QUANTITATIVE LIBS

\section{Introduction}

Laser-induced breakdown spectroscopy (LIBS) is an analytical technique that uses atomic emission to identify and quantify the elemental composition of a sample. LIBS enjoys several advantages, including the ability to make in situ and remote measurements, little or no need for sample preparation, applicability to solid, liquid, gaseous, and aerosol samples, simultaneous measurement of multiple elements, and very short processing times. This makes LIBS the measurement system of choice in many applications, such as process monitoring ${ }^{[1]}$, remote sensing ${ }^{[2]}$, biomedical applications ${ }^{[3]}$, and detection in hostile environments, ${ }^{[4]}$ with many other relevant issues and applications reported recently. ${ }^{[5],[6]}$

The LIBS process involves focusing a laser beam to a small focal volume. Within this volume, the sample is rapidly heated, vaporized, and ionized, creating a luminescent plasma characterized by atomic and continuum emission. As the plasma cools, the atoms and molecules within emit light at wavelengths unique to the species present; this light is the source of the LIBS analytical signal. Quantitative LIBS analysis operates on the assumption that the sample is rapidly and completely dissociated and diffused within the highly energetic plasma, resulting in analyte emission at the representative bulk plasma temperature and a signal linear with increased mass concentration. However, many studies have shown these assumptions do not always hold. ${ }^{[7],[8],[9],[10],[11],[12]}$ As explained in previous work, ${ }^{[8]}$ the validity of those assumptions is dependent on process rates of heat and mass transfer. 
To that end, Hohreiter and Hahn ${ }^{[13]}$ used quantitative plasma imaging of particlederived calcium atoms to measure the diffusion timescales. Their results show that the diffusion timescale is on the same order of magnitude as plasma emission, namely about $15 \mu \mathrm{s}$ for the vaporization of $2 \mu \mathrm{m}$ glass particles. During the early residence time, calcium emission originated exclusively from an area surrounding the location of the original particle and slowly diffused outward during the analysis. This result is in agreement with Lithgow and Buckley, ${ }^{[14]}$ who found spatially inhomogeneous atomic emission in magnesium containing aerosols, and beyond LIBS, by Hieftje et al. ${ }^{[15]}$ who previously proposed a diffusion process in analytical flames and plasmas.

In aggregate, these previous studies suggest that the rate of heat transfer from the plasma to the particle (dissociation), the mass transfer rate of the atomic species (mass diffusion), and the radiative decay rate of the plasma (plasma cooling) are all on the same time-scale as typical LIBS atomic emission analysis; as such, the idealized nearinstantaneous behavior, as described earlier, cannot be assumed in a LIBS plasma.

Indeed, Dalyandar et al. ${ }^{[16]}$ calculated the dimensionless Lewis number, $L e=\frac{\alpha}{D}$, relating thermal diffusivity, $\alpha$, to the mass diffusivity, $D$, during a numerical simulation and found it to be on the order of unity, corroborating the comparable time-scales of heat and mass transfer in laser-induced plasmas.

In light of these results, Diwakar et al. ${ }^{[17]}$ used magnesium-cadmium particles to calculate the emission temperature of atoms versus delay time, and found the temperature to increase with time over a certain early time regime. Given the localized nature of aerosol particle-derived emissions, as stated above, and the fact that bulk plasma temperatures decrease rapidly with time, it was concluded that the temperatures 
measured were local plasma temperatures immediately surrounding the particle (i.e. $\mu \mathrm{m}$ to $\mathrm{mm}$ length scales). The authors theorize that in order to dissociate, the particle draws a large amount of energy from the immediately surrounding plasma. Since the rate of heat transfer within the plasma is on the same timescale as the particle dissociation, the heat lost by the plasma to the particle is not rapidly replaced, creating a localized perturbation of the plasma around the dissociated atoms and causing the analyte atoms to emit at an altered effective temperature. As the atoms diffuse to the hotter parts of the plasma and energy is transferred to the locally-perturbed plasma region from the bulk, the emitting temperature of the atoms begins to change until the particles reach an equilibrium temperature with the bulk plasma.

These results are consistent with measurements done by Diwakar et al. ${ }^{[18]}$ who found inflections in the slopes of the temperature curve of Lu and the ion-to-neutral ratio of $\mathrm{Mg}$ at around the 15-20 $\mu$ s point of plasma evolution. Miclea et al. ${ }^{[19]}$ and Groh et al. ${ }^{[20]}$ found similar results in an inductively-coupled plasma, demonstrating the importance of mass transfer in ICP systems.

The goal of the current study is to further explore the bulk and local plasma temperature and electron density, using gaseous analytes and particulate metals as possible within the plasma, respectively, in order to observe any temperature differences and locate the point of equilibrium within the plasma. An additional goal is the further understanding of the presence of large quantities of a matrix element within the aerosol particles and whether the presence additionally alters the local plasma conditions. 


\section{Experimental Methods}

The system used for this experiment is similar to that reported previously[21] and is shown in Figure 1. Briefly, a 1064-nm Q-switched Nd:YAG laser operating with 300$\mathrm{mJ}$ pulse energy, 10-ns pulse width, and $5-\mathrm{Hz}$ pulse repetition rate was used as the plasma source. An expanded, 12-mm diameter beam was focused inside a six-way vacuum cross using a 75-mm UV grade plano-convex lens. The plasma emission was collected along the incident beam in a backward direction and separated using a 50-mm elliptical pierced mirror. The collected plasma emission was launched into an optical fiber bundle, coupled to a spectrometer (2400-grove/mm grating, 0.12-nm optical resolution) and recorded with an intensified charge-coupled device (iCCD) array. Neutral density (ND) filters were used as necessary to prevent saturation of the detector. 


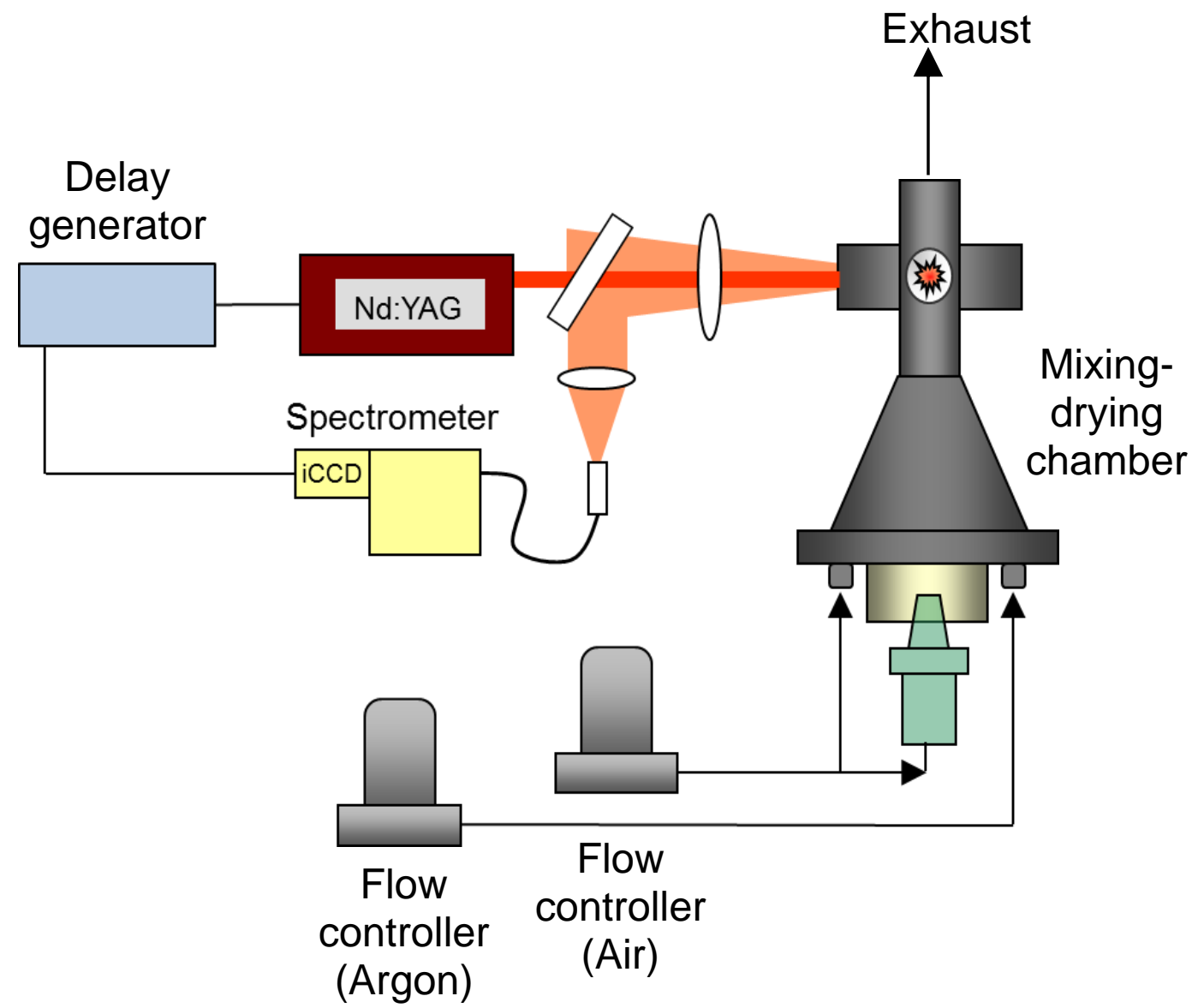

Figure 1. Experimental apparatus used for present work (not to scale).

Argon, nitrogen, and water vapor (i.e. source of hydrogen) were used for the bulk plasma analysis. For water, ultra-purified deionized water was nebulized with $5 \mathrm{lpm}$ of purified, dry air. This hydrogen nebulization stream was mixed in a co-flow of argon and purified, dry air at a rate of $21 \mathrm{lpm}$ each. For the aerosol particle generation, the nebulized water was replaced with an aqueous solution of aluminum, manganese and lutetium (SPEX CertiPrep ICP-grade). Specifically, $10,000 \mu \mathrm{g} / \mathrm{mL}$ solutions of aluminum and manganese were each diluted down to $2,000 \mu \mathrm{g} / \mathrm{mL}$ with deionized water and 
mixed, in equal amounts, with $1,000 \mu \mathrm{g} / \mathrm{mL}$ of lutetium, resulting in the final mixture with concentrations of $667 \mu \mathrm{g} / \mathrm{mL}$ of both aluminum and manganese, and $333 \mu \mathrm{g} / \mathrm{mL}$ of lutetium. In the sodium matrix experiments, $10,000 \mu \mathrm{g} / \mathrm{mL}$ of ICP-grade sodium was added in equal amounts to the above mentioned solutions, resulting in a mixture containing $500 \mu \mathrm{g} / \mathrm{mL}$ aluminum and manganese, $250 \mu \mathrm{g} / \mathrm{mL}$ lutetium, and 2,500 $\mu \mathrm{g} / \mathrm{mL}$ sodium.

Transmission electron microscopy (TEM) was performed on a sample of aerosol particles gathered from the sample chamber. The presence of roughly spherical particles between approximately $50 \mathrm{~nm}$ and $1 \mu \mathrm{m}$ was confirmed, as seen in Figure 2 . Energy-Dispersive X-ray Spectroscopy (EDS) confirmed the presence of $\mathrm{Al}, \mathrm{Lu}, \mathrm{Mn}$, and $\mathrm{Na}$ within individual particles; a representative EDS spectrum is shown in Figure 2. These tests confirm that the aerosol stream, and by extension the plasma, contains sub-micron, multi-element aerosol particles; it is also noted that the particle sizes are well below published limits for complete dissociation in laser-induced plasmas ${ }^{[8], ~[9], ~[10] ~}$ 


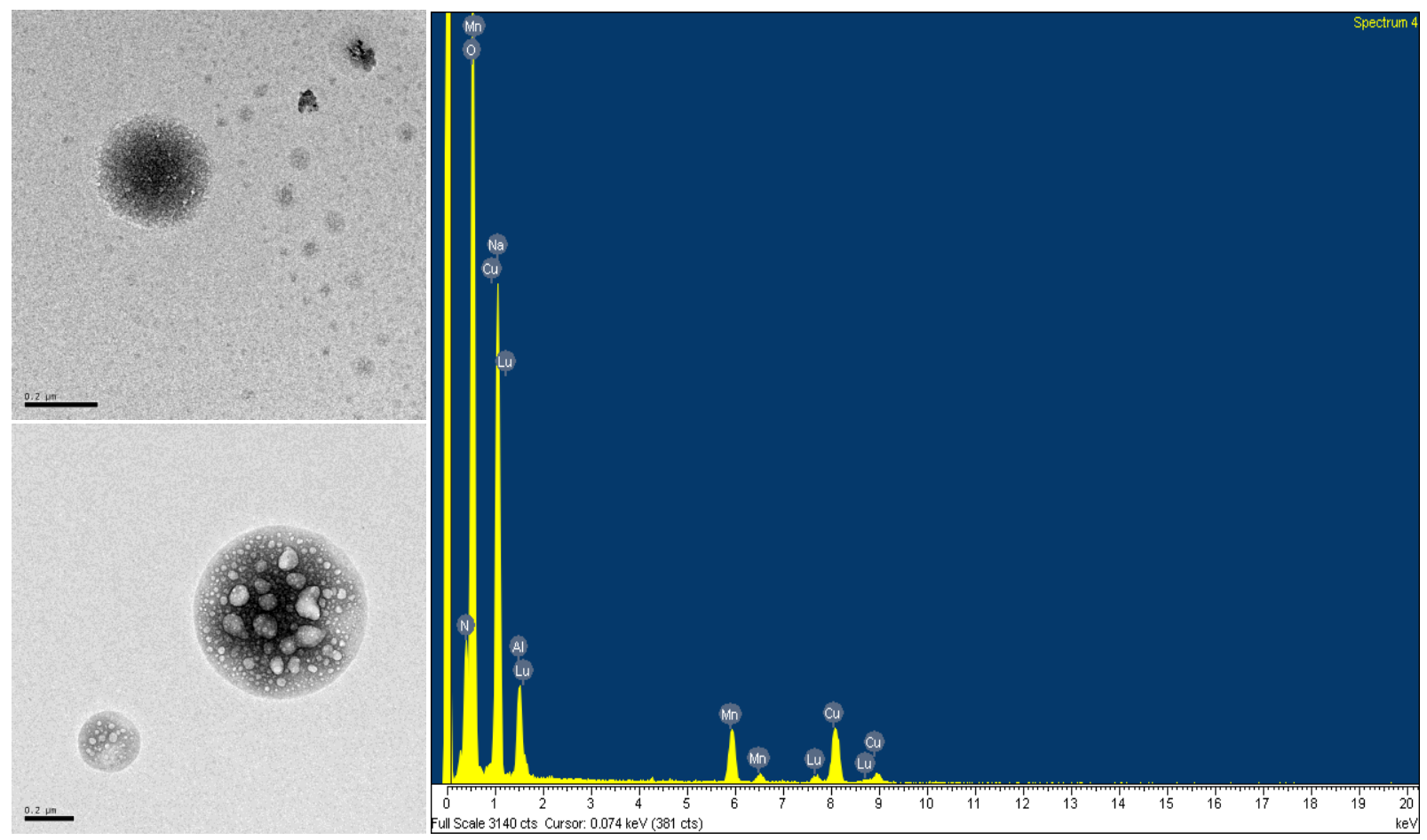

Figure 2. Sample TEM images of aerosol particles formed in aerosol stream during particulate analysis (left) and sample EDS spectrum for the sodium matrix experiments (right). EDS confirms the presence of multi-element aerosols, with all four elemental matrix components present in single particles. Copper is from the TEM grid.

Eight different spectral windows were used throughout the study to capture the emission lines of the various elements. For the purely gaseous plasma, four windows were used, centered about 415, 480, 500, and $656 \mathrm{~nm}$. For the aerosol measurements, including aerosol in the sodium matrix, four additional windows were used, centered about $260,310,350$, and $400 \mathrm{~nm}$. In each window, spectra were collected in a range of temporal delays between 0.1 and $250 \mu \mathrm{s}$; however, not all delays were used in each window (see Table 1). Specifically, the delays used were $0.1,0.2,0.3,0.4,0.5,0.75$, $1.0,1.5,2.0,3.0,5.0,7.5,10,15,25,40,65,100,150$, and $250 \mu$ s. At each delay, six 
sets of data were collected, over multiple days, with each set comprising the average of 600 different plasma events (i.e. laser shots).

Table 1. Range of delays used in each spectral window

\begin{tabular}{cl}
\hline $\begin{array}{l}\text { Spectral window center } \\
\text { wavelength }(\mathrm{nm})\end{array}$ & Temporal range $(\mu \mathrm{s})$ \\
\hline 260 & $0.5-150$ \\
310 & $0.1-250$ \\
350 & $0.1-100$ \\
400 & $0.1-150$ \\
415 & $0.1-10$ \\
480 & $0.1-10$ \\
500 & $0.1-5$ \\
656 & $1.0-40$ \\
\hline
\end{tabular}

\section{Intensity Normalization}

A calibrated lamp source (1000-W tungsten halogen, $250-2400 \mathrm{~nm}$ ) was used to correct for the relative spectrometer spectral response. At each wavelength used, the ratio of the measured signal from the lamp (in arbitrary units) to the actual irradiance, as given by the calibration equation, was calculated and used as a correction factor. This resulted in an irradiance correction factor at each wavelength that was then applied to all the spectra gathered, thereby providing a common intensity scale across all wavelengths.

Because the spectral intensity of the calibrated lamp source was much weaker at lower wavelengths, a stray light correction was performed for the 260 and $310 \mathrm{~nm}$ windows before their irradiance correction factors were calculated. The measured signals at these windows using a $355 \mathrm{~nm}$ high-pass filter (i.e. noise) were compared to 
those without the high-pass filter (i.e. signal + noise) with the true signal taken as the difference between the two. Using the above process, all spectral windows were corrected to the same relative intensity scale. Finally, ND filters were used and adjusted as necessary to avoid saturation of the iCCD.

\section{Plasma Diagnostics}

Once all the above corrections were applied and all data brought to the same relative scale, the plasma excitation temperature was calculated using the Boltzmann plot method ${ }^{[4]}$ for gaseous species (Ar, N and $\mathrm{H}$ ), assumed as corresponding to bulk plasma conditions, and particulate-derived analyte species ( $\mathrm{Lu}, \mathrm{Mn}, \mathrm{Al})$, assumed as corresponding to local plasma conditions. See Figure 3 for representative spectra. It is noted that hydrogen (from water desolvation) is considered as representative of the bulk plasma due to the essentially complete evaporation of the nebulized droplets prior to entering the sample chamber, hence water vapor, as noted previously ${ }^{[16]}$. Since the same relative intensity scale was determined for all wavelength regions, Boltzmann plots using lines from different spectral windows were utilized. Overall, temperatures were calculated using Ar II, N II, N I, Lu II, Mn II and Mn I. 


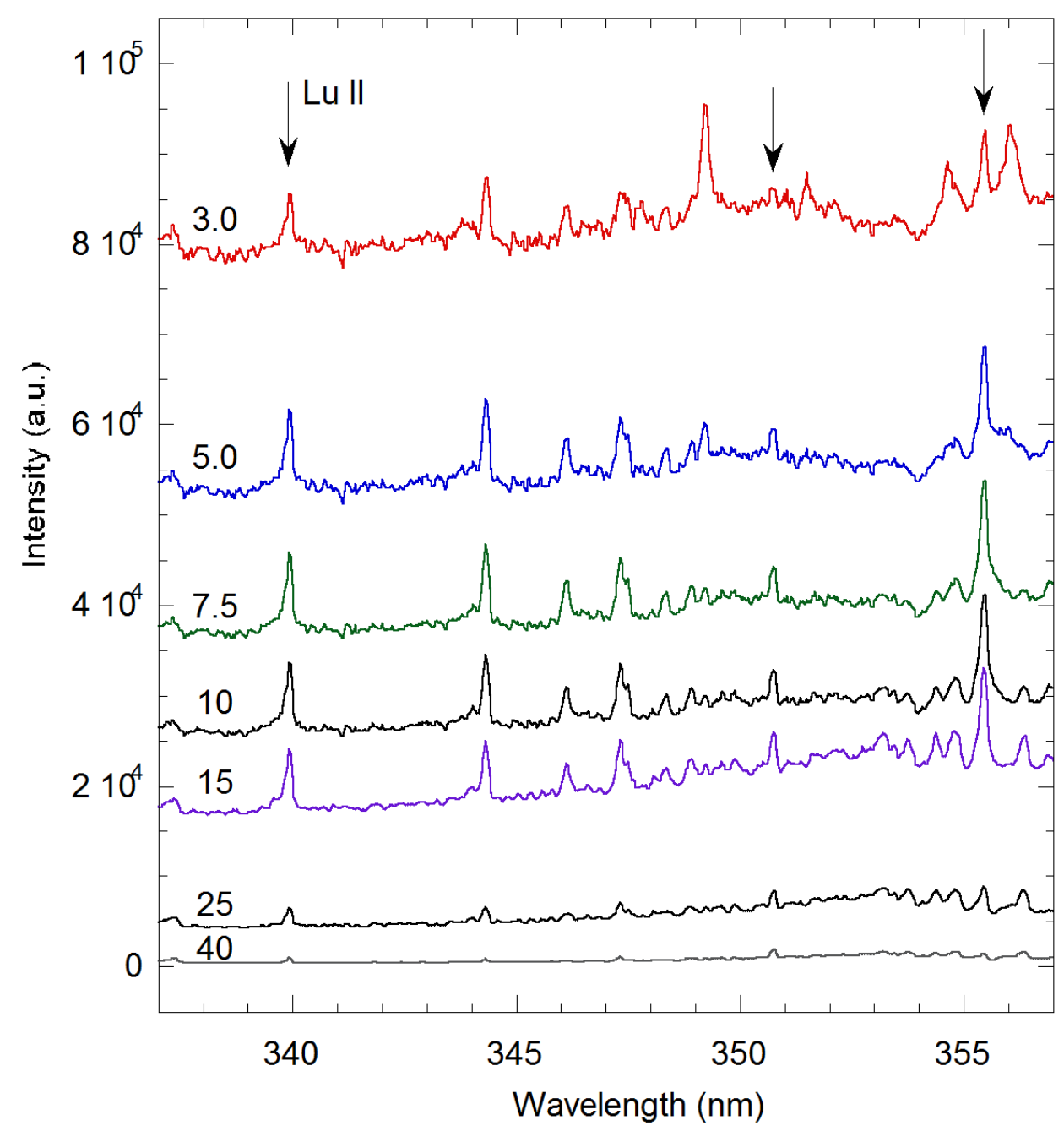

Figure 3. Sample spectra showing the three Lu II lines used for temperature calculation. Delay times with respect to breakdown initiation are shown in microseconds. Arrows denote the Lu II emission lines.

Electron densities were also calculated using Stark broadening,${ }^{[4]}$ for $\mathrm{H}$ alpha and Ar II, with representative hydrogen data shown in Figure 4. A low-pressure mercuryargon lamp (Ocean Optics, model HG-1) was used to find the instrumental broadening width, which was then used to correct the measured widths (at full width half-maximum) to obtain an approximation of the Stark broadening width. For the $\mathrm{H}$ alpha electron density calculation, a series of Stark widths $(\mathrm{nm})$ was calculated for electron densities of 
$1 \mathrm{E} 15$ to $1 \mathrm{E} 18 \mathrm{~cm}^{-3}$ using Griem's theory. ${ }^{[22]}$ From that data, a plot of $(\Delta \lambda)^{3 / 2} v n_{e}$ was drawn and a linear fit applied according to Griem's equation for linear Stark effect: ${ }^{[23],[24]}$

$$
\begin{aligned}
& \Delta \lambda_{F W H M}=2 \alpha_{1 / 2}(2 \pi)(4 / 15)^{2 / 3} e\left(n_{e}\right)^{2 / 3} \\
& \Rightarrow n_{e}=C_{1}\left(n_{e}, T\right) \Delta \lambda_{F W H M}^{3 / 2}
\end{aligned}
$$

where $\alpha_{1 / 2}$ is the fractional half-width of the reduced Stark profile, $e$ is the electron charge (4.8032E-10 esu), and $\mathrm{C}_{1}$ is the slope of the linear fit which is weakly dependent on electron density and temperature. This process was done for temperatures of 10,000 $\mathrm{K}$ and $20,000 \mathrm{~K}$. The $\mathrm{H}$ alpha electron density calculated from the two different fits was then averaged to yield the reported electron density.

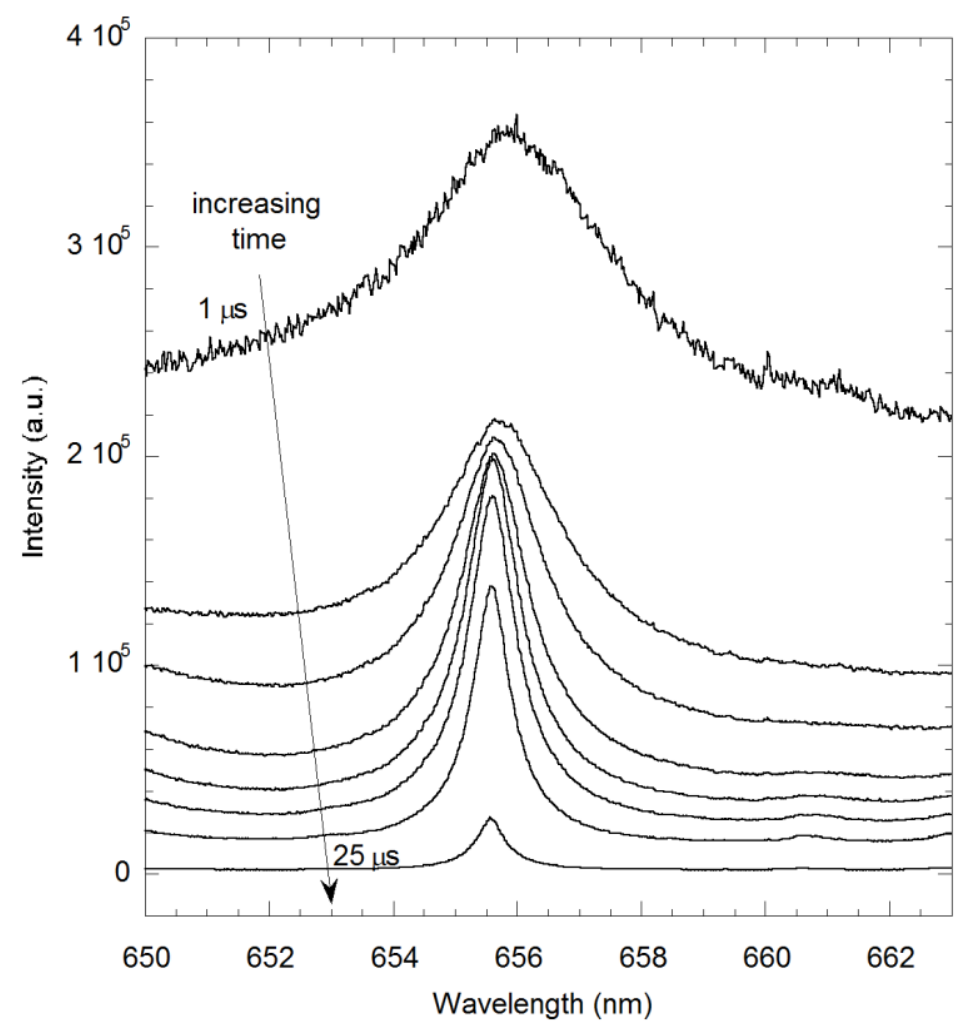

Figure 4. Sample spectra showing the $\mathrm{H}$ alpha line at various delay times following breakdown in the non-sodium aerosol matrix. 
For the electron density calculations using argon, the Stark broadening equation given by Corsi et. al. was used: ${ }^{[4]}$

$$
\begin{aligned}
& \Delta \lambda_{F W H M}=2 w\left(\frac{n_{e}}{10^{16}}\right) \\
& \Rightarrow n_{e}=C_{2}\left(n_{e}, T\right) \Delta \lambda_{F W H M}
\end{aligned}
$$

Using published values for the Stark broadening width and electron density at different temperatures, between 10,880 and $13,880 \mathrm{~K},{ }^{[25]} \mathrm{C}_{2}$ was determined at each temperature; the $C_{2}$ values were then averaged, resulting in a value of $3.67 \mathrm{E} 18 \mathrm{~cm}^{-3}$ $\mathrm{nm}^{-1}$, and used to determine the Ar II electron density. The various lines used to calculate electron temperature and density are shown below in Table 2. 
Table 2. Elemental lines used for excitation temperature and electron density calculations, along with their respective spectral windows and the properties each line was used to determine.

\begin{tabular}{|c|c|c|c|}
\hline Element & Lines (nm) & Spectral windows (nm) & Properties determined \\
\hline $\mathrm{Al} \mathrm{I}$ & $\begin{array}{r}308.21,309.27,309.28 \\
394.4,396.15\end{array}$ & 310,400 & $\mathrm{~T}_{\mathrm{e}}$ \\
\hline \multirow{2}{*}{ Ar II } & $\begin{array}{c}354.56,355.95,385.06, \\
392.86,487.99,506.20\end{array}$ & $350,400,480,500$ & $\mathrm{~T}_{\mathrm{e}}$ \\
\hline & 484.78 & 480 & $\mathrm{n}_{\mathrm{e}}$ \\
\hline H I & 656.28 & 656 & $\mathrm{n}_{\mathrm{e}}$ \\
\hline Lu II & $339.71,350.74,355.44$ & 350 & $\mathrm{~T}_{\mathrm{e}}$ \\
\hline Mn II & $\begin{array}{r}259.37,260.57,293.31 \\
293.93,344.20,346.03\end{array}$ & $260,310,350$ & $\mathrm{~T}_{\mathrm{e}}$ \\
\hline Mn I & $\begin{array}{r}403.08,403.31,403.45 \\
404.14,404.87\end{array}$ & 400 & $\mathrm{~T}_{\mathrm{e}}$ \\
\hline N II & $343.71,504.51$ & 350,500 & $\mathrm{~T}_{\mathrm{e}}$ \\
\hline N I & $\begin{array}{r}413.76,414.34,415.15 \\
467.07\end{array}$ & 415,480 & $\mathrm{~T}_{\mathrm{e}}$ \\
\hline
\end{tabular}

\section{Results and Discussion}

Once all irradiance corrections were applied as described above, all spectral windows were of the same relative intensity scale, as shown in Figure 5. Of note are the overlapping windows at about $400-415 \mathrm{~nm}$ and $480-500 \mathrm{~nm}$, which show excellent intensity agreement with one another. 


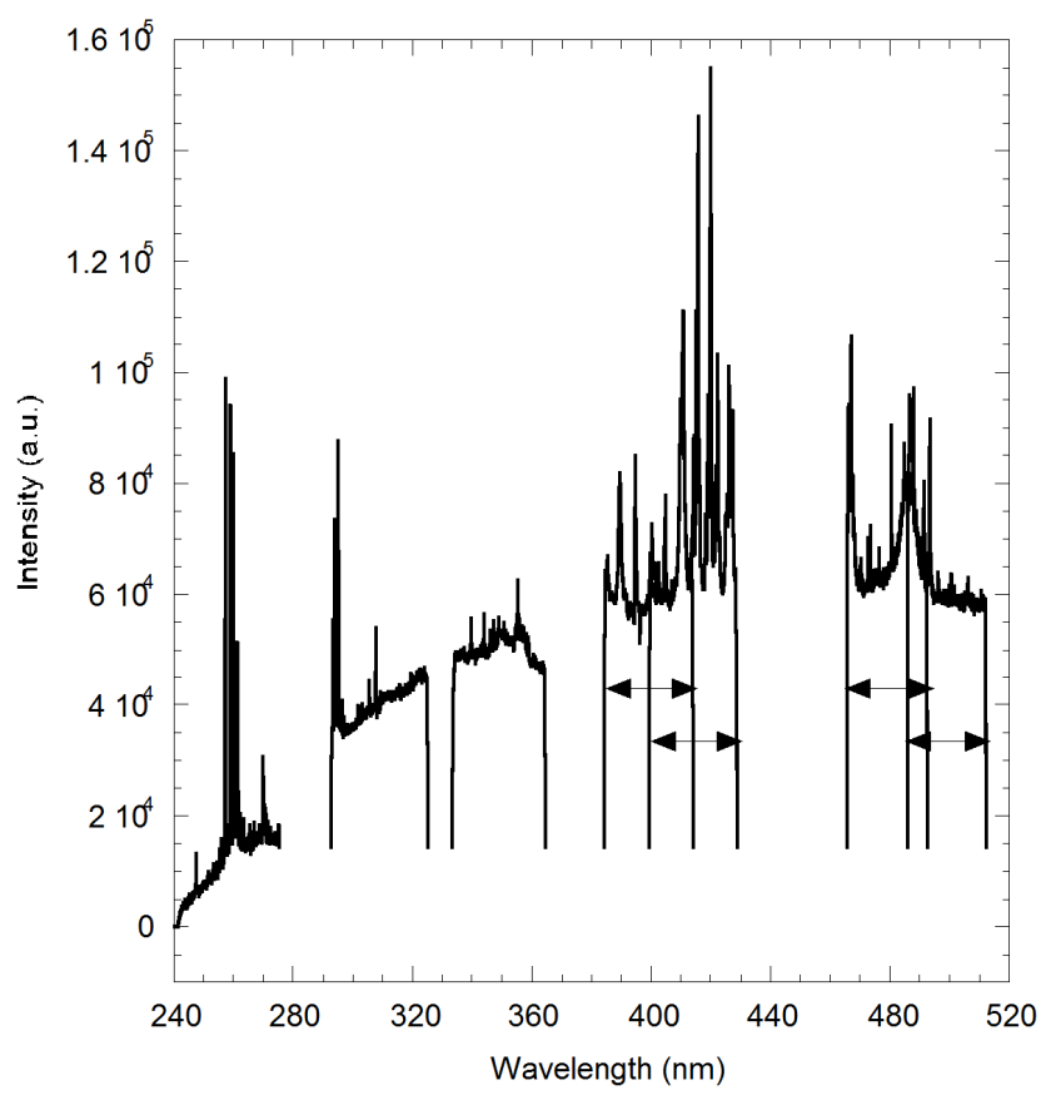

Figure 5. Spectral data at a delay of $5 \mu$ s after irradiance corrections have been applied. For instances of overlapping windows, the arrows denote the limits of the individual spectral windows.

From the relative spectral data, the intensities (full peak integration above continuum) of the lines defined above in Table 2 were calculated; Boltzmann plots were then generated from these intensities. The number of peaks used for these plots varied between 2, for N II only, and 6; the R values for the linear fits were mostly 0.98 or greater, with all but one fit $(R=.81)$ having an $R$ value of at least 0.9 .

From these Boltzmann plots the plasma excitation temperature was derived for the aforementioned species. The temperature plot from Ar II, displayed in Figure 6 below, exhibited some oscillation in early times (within the first $1 \mu \mathrm{s}$ ), but remained relatively flat to about $3 \mu \mathrm{s}$. The N II temperature, also displayed in Figure 6, showed similar 
oscillatory behavior at early times, but with an overall trend of decrease by its termination at $1 \mu \mathrm{s}$; both showed temperatures around 10,000 K. The temperature oscillation observed with both species is well-correlated, which suggests some interaction with the plasma expansion (e.g. internal shock reflection) as observed with previous modeling efforts. ${ }^{[26]}$

In contrast, the temperature derived from N I data (see Figure 8 below) showed a monotonic exponential decay over the range from 3-10 $\mu \mathrm{s}$, but with temperatures around $25,000 \mathrm{~K}$, which is much higher than those derived from Ar II and N II. The observed N II lines were unresolved beyond $1 \mu$ s due to interference; the same applies to Ar II lines beyond $3 \mu \mathrm{s}$. Ar I were either unresolved or subject to interference at all time-scales, and as a result, they were unsuitable for use in the Boltzmann plots and subsequent temperature calculations.

Figure 6 also shows representative error bars for the temperature data of N II and Ar II lines. These error bars were determined via a Monte Carlo scheme by calculating the $\ln (\lambda \mathrm{I} / \mathrm{gA})$ values of the Boltzmann plot for all six sets of data as well as that of their average spectrum. From this data, the standard deviation was also calculated. It was then assumed that the values follow a normal distribution centered about the value calculated from the average spectrum, and with a standard deviation equal to the one calculated. For each delay time, a value for each peak used in the Boltzmann plot was then selected at random, with the probability of selection equal to that of a normal distribution, and the slope of the plot calculated; this process was repeated five thousand times. The average slope of all five thousand calculations was very close to the slope determined using the averaged spectrum, indicating a sufficiently large 
sampling population. The standard deviation of the population was then assumed to be equal to the standard deviation of the slopes of the Boltzmann plots.

The temperature was then calculated using the slope plus one standard deviation and the slope minus one standard deviation and the difference between the two calculated $(2 \sigma)$. This difference ranges between $2 \%$ and $56 \%$ of the average temperature. The error bars in the temperature data represent $\pm 1 \sigma$ of the average temperature derived from all six data sets.

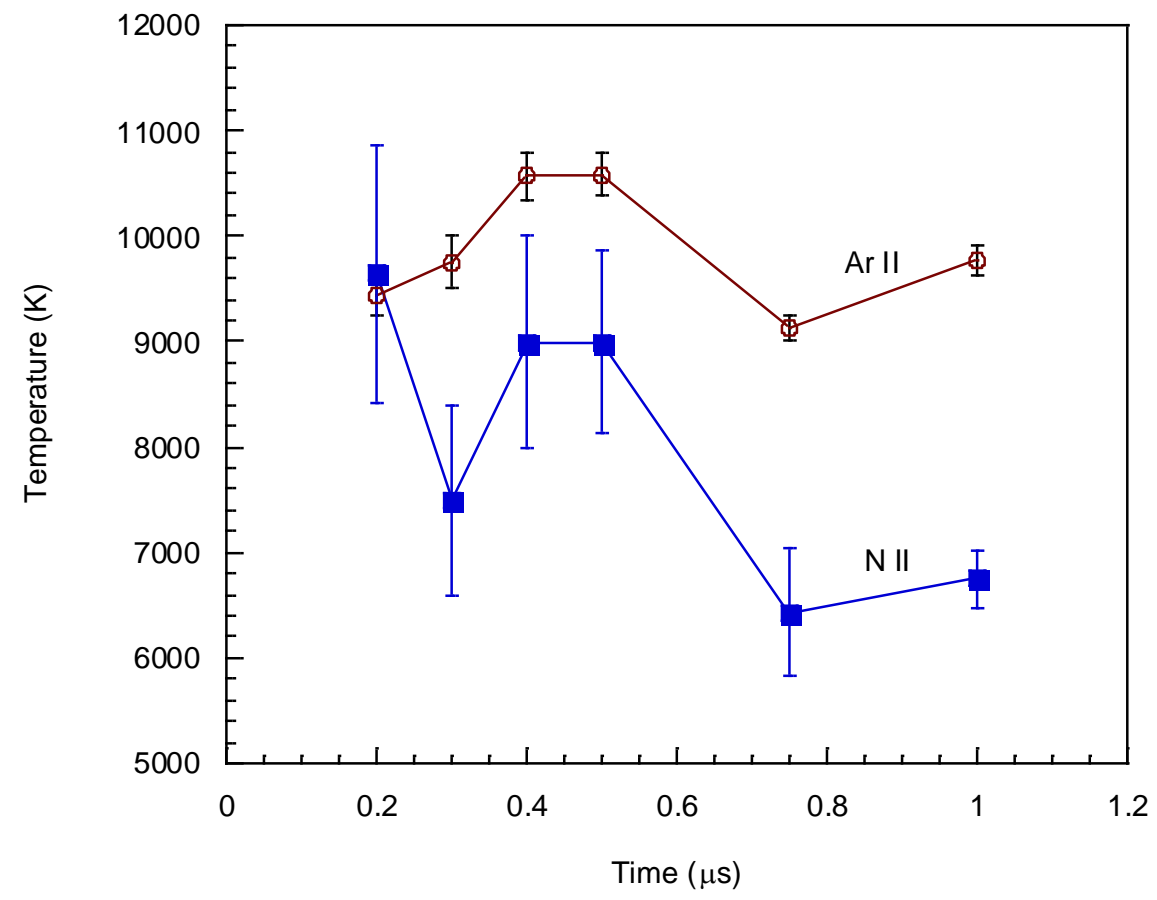

Figure 6. Bulk plasma temperatures as a function of plama decay time as calculated from Ar II and N II.

For the particulate-derived species, the emission lines were resolved over a longer time period, up to $100 \mu \mathrm{s}$. The temperatures calculated were generally found to decrease with plasma decay time; however, the rate of decrease of the plots is not 
constant. In the Lu II temperature plot, shown in Figure 7, the rate of cooling is almost linear between 5 and $25 \mu$ s, at which point the plot adopts an exponential rate of decrease. The same trend is seen in the Mn II data, also in Figure 7, in which the slope is nearly flat between 2 and $15 \mu \mathrm{s}$, but then gives way to an exponential decay. It is noted that the Lu atoms are markedly heavier than $\mathrm{Mn}$ (175.0 vs. $54.9 \mathrm{amu})$, leading to an expected difference in mass diffusion coefficient and therefore, potential differences in spatial distribution. Overall, the temporal behavior of these two analyte elements is in very good agreement with that seen in previous studies. ${ }^{[17],[18],[19],[20]}$

This change in the slopes of the temperature curves is attributed to heat from the bulk plasma diffusing into the locally cooled plasma surrounding the emitting analyte atoms, thereby slowing the rate of net energy loss to the surroundings. The $\mathrm{Mn} \mathrm{I}$ temperature data were first recorded at $25 \mu \mathrm{s}$, after this slope shift phenomenon is observed. As a result, it shows no slope shift but does show a monotonic, exponential decay in temperature as is expected. Overall, the values of the temperature are in good agreement with each other for all three particulate-derived species. 

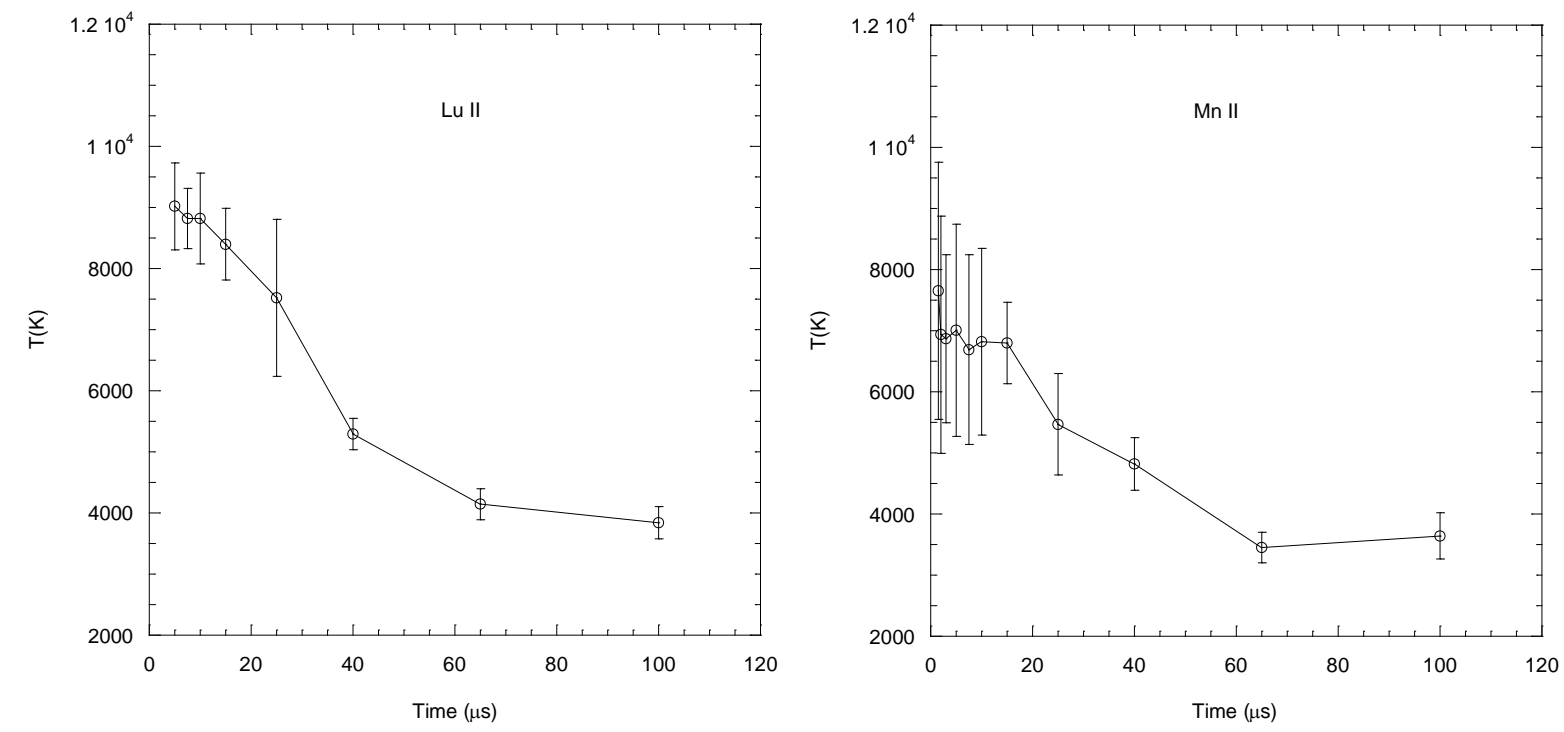

Figure 7. Local plasma temperature as determined by Lu II (left) and Mn II (right) with representative error bars $( \pm 1 \sigma)$.

Figure Figure 8 below shows all the temperature data on the same plot for the gaseous and particulate-derived analyte species. There are multiple phenomena of interest that can be observed. The first is that the temperatures calculated from N I are much higher than all other temperatures observed, as noted above. One might expect the background gas of the neutral $\mathrm{N}$ to be characterized by a larger temperature, as it fills the plasma region away from the particle-associated processes of atomization and 
ionization. It is additionally considered that spectral interference or possibly uncertainty in the spectroscopic constants may play a role, making definitive conclusions about the $\mathrm{N}$ I temperature inconclusive.

Secondly, there is rather good agreement in temperature at the intersection of the gaseous and particulate data, at around 1-10 $\mu$ s. Thirdly, at early times the temperature calculated from Mn II is lower than that from Lu II; this may be due to the differences in the ionization energies of the two elements or differences in the diffusion coefficients as noted above, with the latter parameter giving rise to different spatial-temperature profiles. Manganese, with a first ionization energy of $717.3 \mathrm{~kJ} \cdot \mathrm{mol}^{-1}$, requires more energy to ionize than lutetium, which has a first ionization energy of $523.5 \mathrm{~kJ} \cdot \mathrm{mol}^{-1}$. This difference in ionization energies can result in a greater local cooling effect for manganese compared to the lutetium, thereby lowering the observed temperature. The final item of note is that the temperatures calculated from all three particulate species seem to converge at very long times. This suggests that over time, the different degrees of cooling (trend lines are added as a guide) experienced by each species become negated as the locally cooled regions absorb energy from the bulk plasma. In combination with diffusion throughout the plasma, overall species equilibrium is approached, causing all species to emit at the same bulk plasma temperature in the idealized case. 


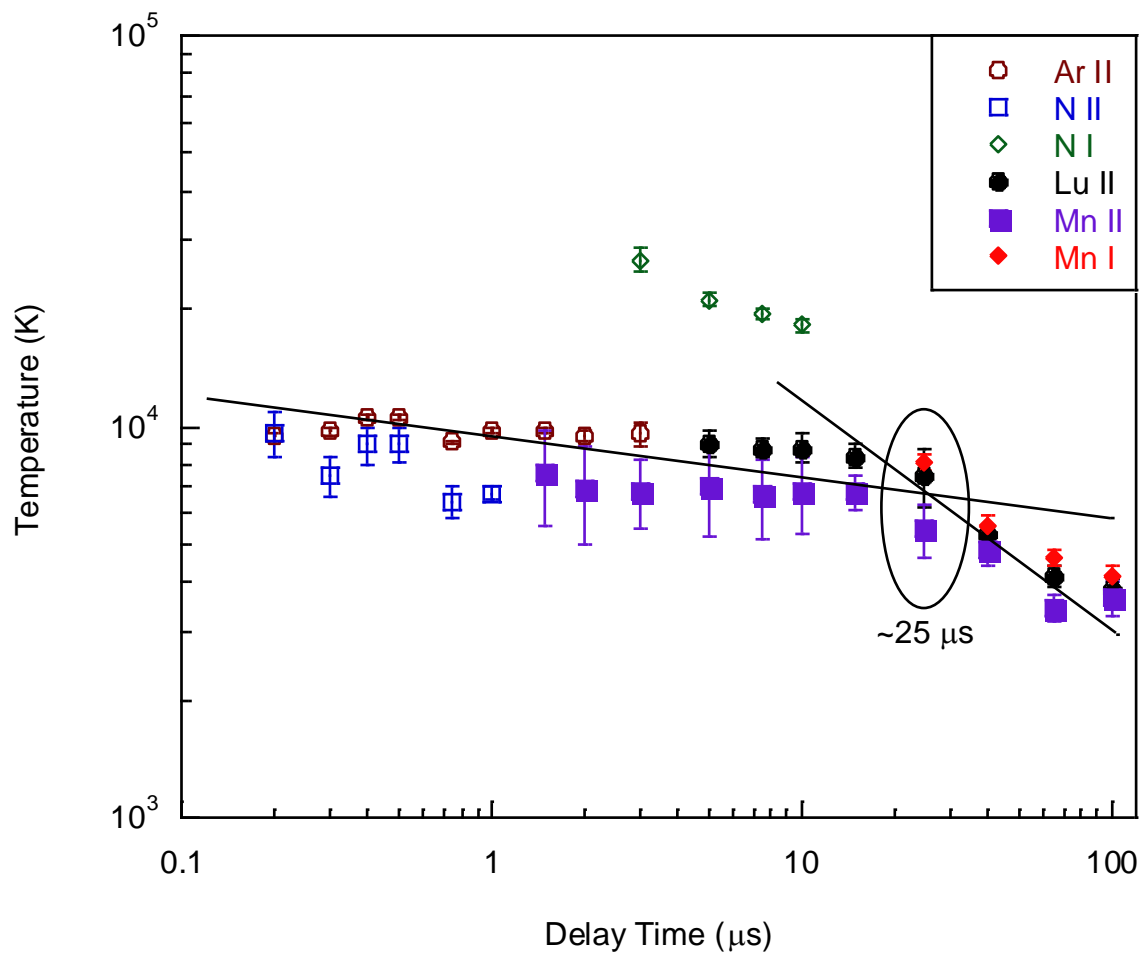

Figure 8. Temperatures derived from all species as a function of plasma delay time. Open symbols refer to gas species (bulk plasma properties) and solid symbols to particulate species (local plasma properties). The shift in slope at about $25 \mu \mathrm{s}$ is indicated with the circle. Representative error bars are included.

It was the intent of this study to directly compare the electron density calculated from the gaseous species to those from the particulate species in a similar manner to the temperature plots above. However, the line widths (FWHM) of the particulatederived species ( $\mathrm{Mn}, \mathrm{Lu}$ and $\mathrm{Al}$ ) were comparable to the instrumental broadening and therefore could not be used for electron density calculations. Figure 9 presents the bulk plasma electron density calculated using the $H_{\alpha}$ line and $\operatorname{Ar}$ II $484.78 \mathrm{~nm}$ line. The two data sets agree extremely well, again corroborating the role of hydrogen and argon as representative of bulk plasma species. 


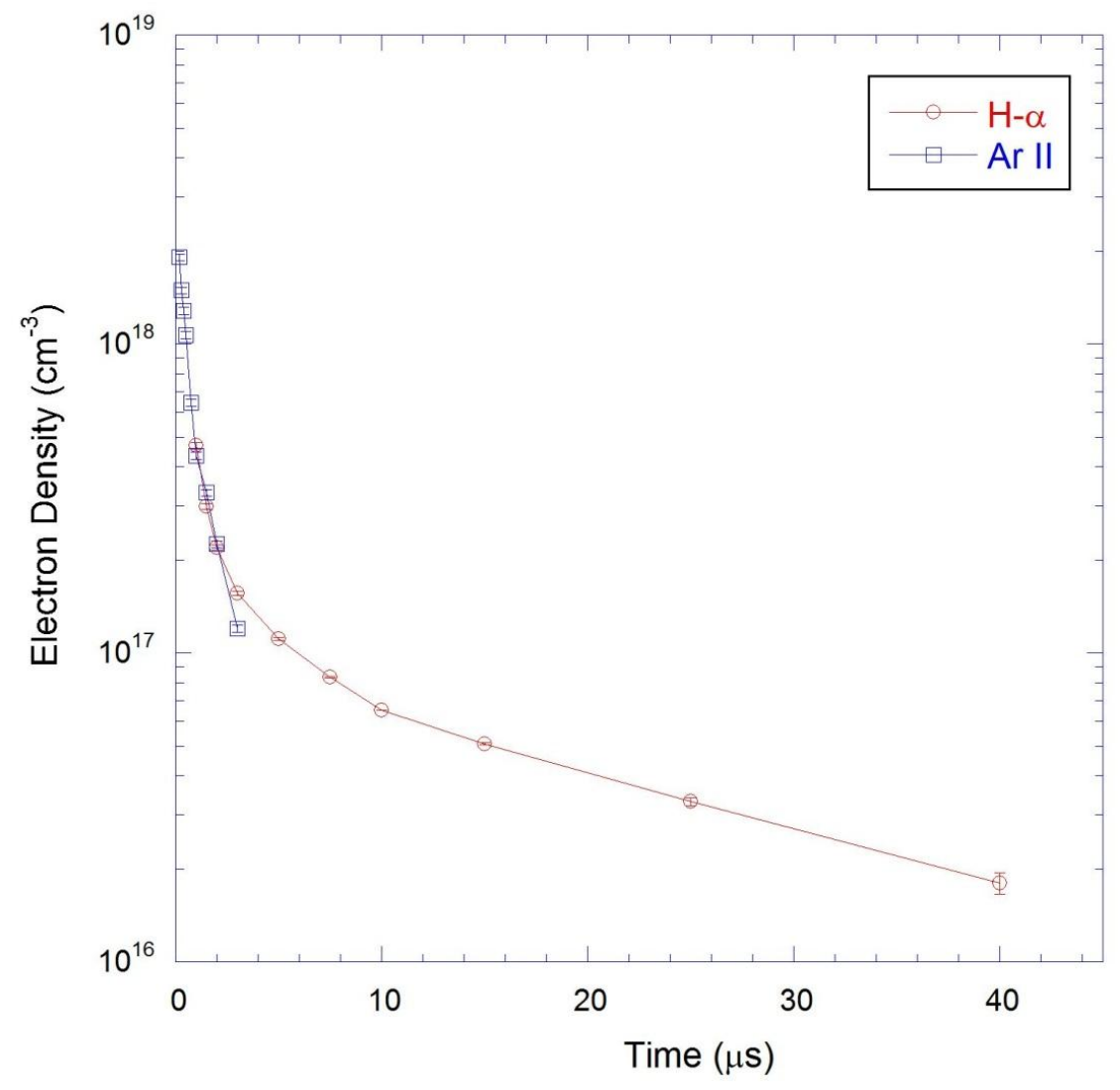

Figure 9. Bulk plasma electron density, as measured by the $\mathrm{H}_{\alpha}$ and $\mathrm{Ar} I \mathrm{I} 484.78 \mathrm{~nm}$ lines, on a semi-log scale. Full error bars denote one standard deviation.

In order to compare the effects of the added sodium matrix on plasma properties, the plasma temperature from Lu II in the Na-free matrix was compared to that in the heavy sodium matrix; the results are shown below in Figure 10. Quantitatively, the two plots fall within the error bars, which makes it difficult to draw statistically significant conclusions from the data available. Qualitatively, the temperatures show two differences. First, there is a slight temperature suppression at early times in the sodium matrix; this may be due to the increase in size and/or number of the particulate species 
due to the presence of additional mass within the plasma volume. This in turn would increase the amount of energy required to vaporize and ionize each particle, leading to the observed temperature suppression.

Secondly, once equilibrium is reached by about $40 \mu \mathrm{s}$, the rate of cooling of the sodium matrix is slower than that of the non-sodium matrix; this can again be attributed to the presence of additional thermal mass (i.e. heat capacity) in the plasma which causes more energy to be present for a given temperature. A more detailed investigation is required to support these findings given the experimental uncertainty.

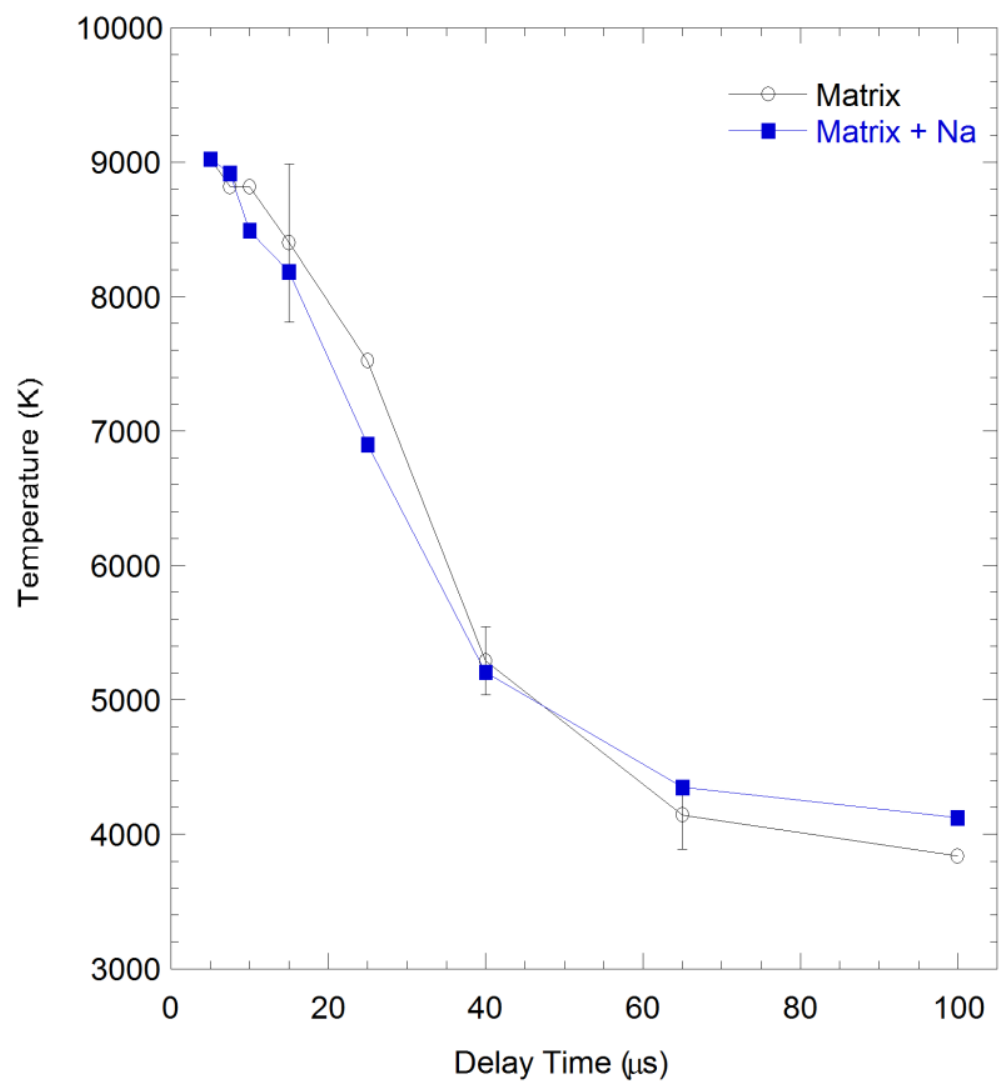

Figure 10. Comparison of the local plasma temperature using Lu II in normal matrix versus the added sodium matrix. Representative error bars are shown $( \pm 1 \sigma)$. 


\section{Conclusions}

The goal of this study was to compare bulk and local plasma properties in order to explore the potential equilibrium time scales. In addition, a preliminary investigation was performed into the effect of large quantities of a matrix aerosol within the plasma on plasma conditions. The data successfully revealed changes in the temperature profile of particle-derived analyte species, confirming the effect of local perturbation of plasma properties. This effect is significant within the first $20-40 \mu$ s of plasma lifetime. The intersection of the bulk, gas species temperature and the local, particle-derived temperature were in general agreement. However, since the gas species temperature values were only measurable at very early plasma lifetimes, it did not provide the desired direct comparison with the particulate-derived species emission on the earliest time-scales. The bulk plasma electron density was successfully calculated, producing excellent agreement between the $\mathrm{Ar}$ and $\mathrm{H}$ data, and leading to the conclusion that strong equilibrium exists between the gaseous species (i.e. bulk plasma). It was found that the matrix of the aerosol particles may affect the extent of perturbation of the local plasma as well as the rate of temperature loss for particle-derived species, although statistical significance was not able to be quantified.

In all, the results of this study corroborate the idea that within a laser-induced plasma, there are finite rates of heat and mass transfer, contrary to the idealization of rapid (i.e. infinite) dissociation and diffusion. This effect is considered a major contributing factor to observed matrix effects in LIBS analysis. However, as shown in this study, sufficiently long delay times, on the order of $20-40 \mu \mathrm{s}$, serve to mitigate these temporal effects. 


\section{Acknowledgements}

This work was supported by the National Science Foundation and the Deutsche Forschungsgemeinschaft through grant CHE-0822469 and DFG Ni 185/38, respectively, as part of the Plasma-Analyte Interaction Working Group (PAIWG), a collaborative effort of the University of Florida, Federal Institute of Materials Research and Testing (BAM) in Berlin, and ISAS at Technical University Dortmund, jointly funded by the NSF and DFG. 


\section{List of References}

[1] R. Noll, H. Bette, A. Brysch, M. Kraushaar, Laser-induced breakdown spectrometry - applications for production control and quality assurance in steel industry, Spectrochimica Acta Part B 56 (2001) 637-649.

[2] A.J. Ball, V. Hohreiter, D.W Hahn, Hydrogen leak detection using laser-induced breakdown spectroscopy, Applied Spectroscopy 59 (2005) 348-353.

[3] P.B. Dixon, D.W. Hahn, On the feasibility of detection and identification of individual bioaerosols using laser-induced breakdown spectroscopy, Analytical Chemistry 77 (2005) 631-638.

[4] M. Corsi, G. Cristoforetti, I. Gornushkin, N. Omenetto, V. Palleschi, B.W. Smith, E. Tognoni, J.D. Winefordner, From sample to signal in laser-induced breakdown spectroscopy: a complex route to quantitative analysis, in: Laser-Induced Breakdown Spectroscopy (LIBS): Fundamentals and Applications, Cambridge University Press New York, New York, 2006, pp 122-170.

[5] D.W. Hahn, N. Omenetto, Laser-induced breakdown spectroscopy (LIBS), Part I: Review of basic diagnostics and plasma-particle interactions: Still-challenging issues within the analytical plasma community, Applied Spectroscopy 64 (2010) 336A-366A.

[6] D.W. Hahn, N. Omenetto, Laser-induced breakdown spectroscopy (LIBS), Part II: Review of instrumental and methodological approaches to material analysis and applications to different fields, Applied Spectroscopy 66 (2012) 347-419.

[7] L.J. Radziemski, T.R. Loree, D.A. Cremers, N.M. Hoffman, (1983). Time-resolved laser-induced breakdown spectrometry of aerosols, Analytical Chemistry, 55, 12461252.

[8] D.W. Hahn, Laser-induced breakdown spectroscopy for analysis of aerosol particles: the path toward quantitative analysis, Spectroscopy 24 (2009) 26-33.

[9] M. Essien, L.J. Radziemski, J. Sneddon, Detection of cadmium, lead and zinc in aerosols by laser-induced breakdown spectrometry, Journal of Analytical Atomic Spectrometry 3 (1988) 985-988.

[10] J.E. Carranza, D.W. Hahn, Assessment of the upper particle size limit for quantitative analysis of aerosols using laser induced breakdown spectroscopy, Analytical Chemistry 74 (2002) 5450-5454.

[11] E. Vors, L. Salmon, Laser-induced breakdown spectroscopy (LIBS) for carbon single shot analysis of micrometer-sized particles, Analytical and Bioanalytical Chemistry 385 (2006) 281-286. 
[12] G. Gallou, J.B Sirven, C. Dutouquet, O. Le Bihan, E. Frejafon, Aerosols analysis by LIBS for monitoring of air pollution by industrial sources, Aerosol Science and Technology 45:8 (2011) 918-926.

[13] V. Hohreiter, D.W. Hahn, Plasma-particle interactions in a laser-induced plasma: implications for laser-induced breakdown spectroscopy, Analytical Chemistry 78 (2006) 1509-1514.

[14] G.A. Lithgow, S.G. Buckley, Effects of focal volume and spatial inhomogeneity on uncertainty in single-aerosol laser-induced breakdown spectroscopy measurements, Applied Physics Letters 87 (2005) 011501.

[15] G.M. Hieftje, R.M. Miller, Y. Pak, E.P. Wittig, Theoretical examination of solute particle vaporization in analytical atomic spectrometry, Analytical Chemistry 59 (1987) 2861-2872.

[16] P.S. Dalyandar, I.B. Gornushkin, D.W. Hahn, Numerical simulation of laser-induced breakdown spectroscopy: Modeling of aerosol analysis with finite diffusion and vaporization effects, Spectrochimica Acta Part B 63 (2008) 293-304.

[17] P.K. Diwakar, P.B. Jackson, D.W. Hahn, The effect of multi-component aerosol particles on quantitative laser-induced breakdown spectroscopy: Consideration of localized matrix effects, Spectrochimica Acta Part B 62 (2007) 1466-1474.

[18] P.K. Diwakar, S. Groh, K. Niemax, D.W. Hahn, Study of analyte dissociation and diffusion in laser-induced plasmas: implications for laser-induced breakdown spectroscopy, Journal of Analytical Atomic Spectrometry 25 (2010) 1921-1930.

[19] M. Miclea, C.C Garcia, I. Exius, H. Lindner, K. Niemax, Spectroscopic monitoring of particle size, transport and composition in laser ablation inductively coupled plasma spectrometry, Spectrochimica Acta Part B 61 (2006) 361-367.

[20] S. Groh, C.C. Garcia, A. Murtazin, V. Horvatic, K. Niemax, Local effects of atomizing analyte droplets on the plasma parameters of the inductively coupled plasma, Spectrochimica Acta Part B 64 (2009) 247-254.

[21] M.E. Asgill, D.W. Hahn, Particle size limits for quantitative aerosol analysis using laser-induced breakdown spectroscopy: temporal considerations, Spectrochimica Acta Part B 64 (2009) 1153-1158.

[22] H.R. Griem, Plasma Spectroscopy, McGraw-Hill, New York, New York, 1964.

[23] V. Hohreiter, J.E. Carranza, D.W. Hahn, Temporal analysis of laser-induced plasma properties as related to laser-induced breakdown spectroscopy, Spectrochimica Acta Part B 59 (2004) 327-333. 
[24] O. Samek, D.C.S. Beddows, J. Kaiser, S.V. Kukhlevsky, M. Liska, H.H. Telle, J. Young, Application of laser-induced breakdown spectroscopy in in situ analysis of liquid samples, Optical Engineering 39 (2000) 2248-2262.

[25] N. Konjevic, A. Lesage, J.R. Fuhr, W.L. Wiese, Experimental Stark widths and shifts for spectral lines of neutral and ionized atoms, Journal of Physical and Chemical Reference Data 31 (2002) 819-927.

[26] A. Ya. Kazakov, I.B. Gornushkin, N. Omenetto, B.W. Smith, J.D. Winefordner, Radiative model of post-breakdown laser-induced plasma expanding into ambient gas, Applied Optics 45 (2006) 2810-2820. 